\title{
Online tools to easily build augmented and virtual reality models of molecules that run right on the web
}

\author{
Fabio Cortés Rodríguez, Matteo Dal Peraro and Luciano A. Abriata
}

Laboratory for Biomolecular Modeling, École Polytechnique Fédérale de Lausanne and Swiss Institute of Bioinformatics, 1015 Lausanne, Switzerland

Running title: Quick molecular graphics in web augmented and virtual reality

Abstract. Several groups developed in the last years augmented and virtual reality (AR/VR) programs and apps to visualize 3D molecules, most rather static, limited in content, and requiring software installs, some even requiring expensive hardware. During the Covid-19 pandemic, our team launched moleculARweb (https://molecularweb.epfl.ch), a website that offers interactive content for chemistry and structural biology education through commodity web-based AR that works on consumer devices like smartphones, tablets and laptops. Among thousands of users, teachers increasingly request more biological macromolecules to be available, a demand that we cannot satisfy individually. Therefore, to allow users to build their own material, we built a web interface where any user can build any online AR experience in few steps starting from a PDB structure or from virtual objects/scenes exported from VMD. The website also returns a WebXR session for viewing and manipulating the model in high-end immersive VR headsets with web browsers, here using the Oculus Quest 2. The tool is accessible at https://molecularweb.epfl.ch/pages/pdb2ar.html. 


\section{Introduction}

Incidentally with the Covid-19 pandemic, we launched during 2020 moleculARweb, a website for chemistry and structural biology education through augmented reality (AR) that works on consumer devices like smartphones, tablets and laptops. ${ }^{1}$ The website (https://molecularweb.epfl.ch/) provides several activities where students and educators can either handle virtual molecules and other chemistry-related entities like electronic orbitals with paper-printed markers to compare them in 3D and even to make them interact, or simply anchor virtual molecules to a flat surface such as a table to inspect them immersively by freely moving around them. Each activity is delivered as a regular webpage, so users do not need to install any programs to use them. They just need to access the web page of interest and enable the webcam. More details about moleculARweb are available in our recent publication ${ }^{1}$ (or its preprint), which focuses more on topics pertinent to chemistry and stresses AR achieved through printed markers, only barely introducing examples of biomolecular assemblies and markerless AR, which are the focus of this work.

moleculARweb quickly got thousands of users, students and teachers, many of them accessing from their homes due to the pandemic (constantly monitored at https://clustrmaps.com/site/1ar2f). Online and inperson surveys showed positive effects on learning, strong engagement by students, and high success rates in making the AR activities work, the latter likely due to the entirely web-based nature of the tools, which facilitates deployment as noticed by multiple works. ${ }^{2,3}$

Since the release of moleculARweb, teachers have been increasingly requesting more AR material, especially for biological macromolecules. As this demand is very hard to satisfy individually, we built a tool that teachers can use to build their own material, also helping us to expedite the development of new activities. This new tool consists in a web interface where users can easily build any online AR or VR experience for the web in few steps and without any knowledge about AR, VR nor programming, starting either from a PDB structure or from virtual objects/scenes exported from the VMD ${ }^{4}$ molecular graphics program. Figure 1 shows a summary of the pipeline from input to output. The input can be either a PDB file of a system prepared by the educator or fetched right from the Protein Data Bank, to be converted by moleculARweb into AR/VRcompatible 3D model, or a ready-to-use 3D model or scene exported from VMD in Wavefront format. The output (received by email) consists of two web-AR and one web-VR modes, that users can share without 
restrictions (for example an educator can share with its students). In the first AR mode the object is attached to a cubic marker printed and folded from paper, just like the cube marker of moleculARweb's Virtual Modeling Kit. From this page the user can launch the second AR mode, where the object is displayed in the space of the user just lying over any flat surface detected. The VR mode works on computers, smartphones and tablets, where the molecules are moved and zoomed with the mouse or touch gestures; or in a VR headset with WebXR-enabled browsers ${ }^{5}$ (we tested the Oculus Quest 2, which costs around 400 EUR as of late 2021) where the user can handle the molecules just with its bare hands. In this short article we describe this tool, accessible at https://molecularweb.epfl.ch/pages/pdb2ar.html, with examples covering the two possible kinds of inputs, which we call input modes 1 (from PDB) and 2 (from premade 3D objects).

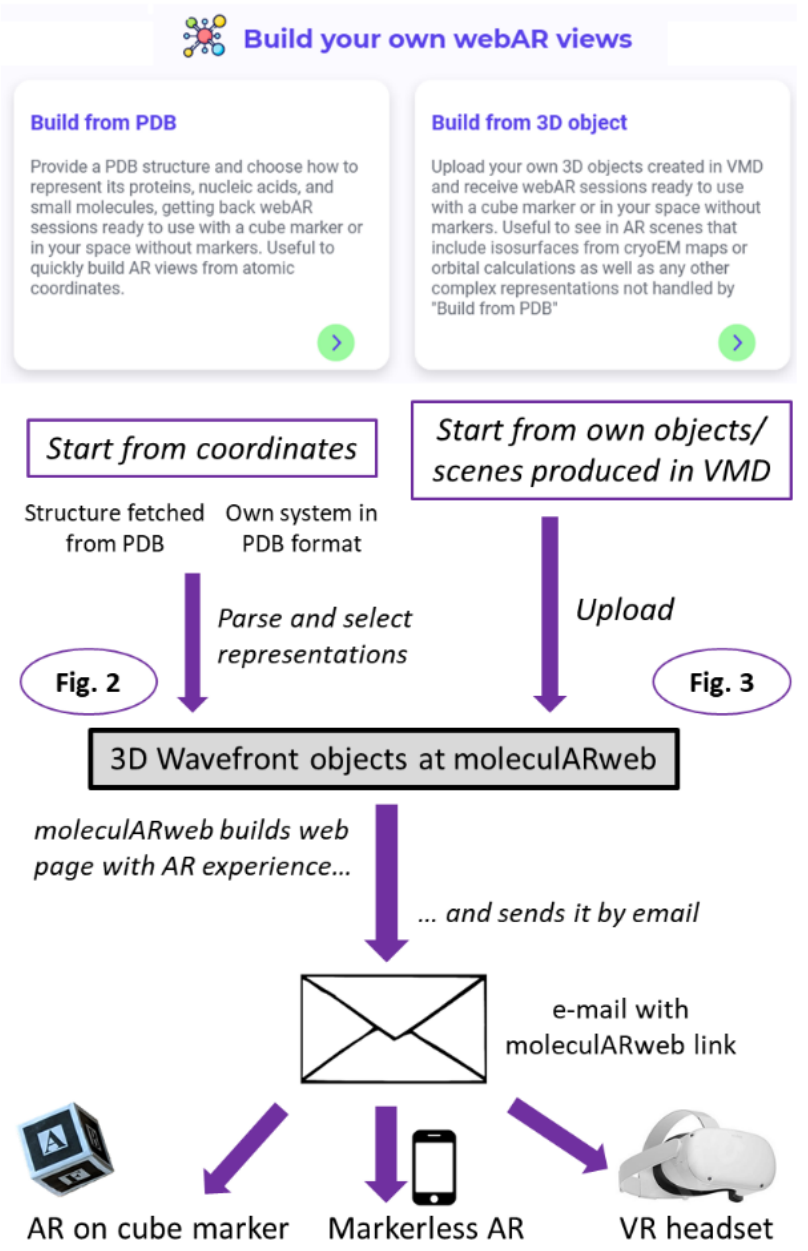

Figure 1. Pipeline for creating customized webAR experiences on molecular systems, starting from coordinates (illustrated in Figure 2) or from premade $\mathrm{VMD}^{4}$ objects or scenes (illustrated in Figure 3 ). The direct link to the tool is https://molecularweb.epfl.ch/pages/pdb2ar.html. The cube marker can be printed and folded from https://molecularweb.epfl.ch/assets/markers/allmarkers.pdf. AR with markers works in all modern browsers and operative systems on smartphones, tablets and desktop computers (user needs to enable webcam use when prompted for the first time); while markerless AR works only in Chrome (best if version 92 or higher) on WebXR-enabled smartphones. In VR headsets the app works inside WebXR-compatible web browsers -we only tested the web browser that comes with the Oculus Quest 2. 


\section{Input mode 1: Atomic coordinates in PDB format}

The first and simplest way to use this new tool is to copy-paste a PDB file of a system prepared by the educator or fetch it directly from the Protein Data Bank using its PDB code, as illustrated in Figure 2 for the protein myoglobin from PDB ID $1 \mathrm{MBN} .{ }^{6}$ Once the PDB file is ready (Figure $2 \mathrm{~A}$ ) the user clicks "Detect molecules" to which the app responds with two lists: one of all protein and nucleic acid molecules detected, and one with all additional special small molecules or "ligands" (Figure 2B). For each entry of these two tables, the user can indicate whether to display it or not, how to display it, and what colors to use (protein and nucleic acid chains are repeated twice in the list to allow multiple representations, such that for example a given protein chain can be displayed as cartoons and also as sticks). Users acquainted with the VMD molecular graphics program will find familiar the terms used in the lists. For example, for $1 \mathrm{MBN}$ one can choose to show protein chain $\mathrm{A}$ as "New cartoon" colored in "Yellow" and then choose to display the HEM group as "Licorice" colored by "Atom name" but highlighting the central iron atom as a sphere ("VDW") colored "Tan".

The next step, after having chosen the wanted representations, is to click "Build VMD script" which will result in a portion of code to be executed at our servers to produce the 3D scenes (Figure 2C). The point of displaying this script in the web page is that users familiar with VMD can edit it to fine-tune the final render.

The "Submit" button at the end of the page runs only if the user provided a title for the activity and an email where to receive the results. A few seconds after clicking this button the user will receive an email with links to the prepared visualizations (Figure 2D). If the view is not as intended the user can make changes right there in the web tool and resubmit to immediately get further emails with the changes applied.

As exemplified in Figure 2E-G for the input based on PDB structure $1 \mathrm{MBN}$, the link delivered by email opens first an AR experience that works with moleculARweb's cube marker (Figure 2E). At the bottom right of this view a button labeled "3D" opens up a view with a white background (Figure 2F) from which a subsequent button launches a markerless AR view where the virtual object lies on a flat surface detected by the device (Figure 2G). The first two modes work in all devices, i.e. smartphones, tablets and computers, while the markerless mode works only on WebXR-enabled smartphones and tablets as it requires the device's pose 
estimation capabilities. This is also valid for inputs prepared from premade VMD scenes, described in the next section.

A

Here we should add a brief text describing the tool and probably some instructions.

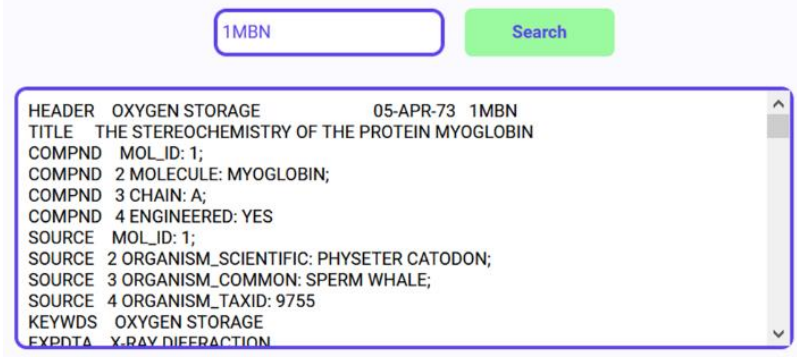

Detect molecules Merge water together

C

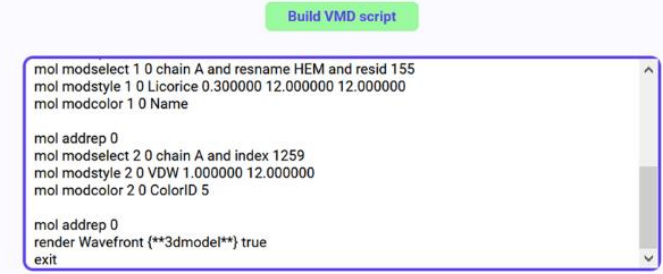
Please add a title for your project and an email address. We will send you the link of the AR experience once it's
ready.

We will not store your email address and it will only be used to send you the link of your project.

$$
\text { Title Myoglobin from PDB 1MBN Email Uuciano.abriata@epf.ch }
$$

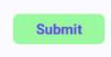

$\mathbf{E}$

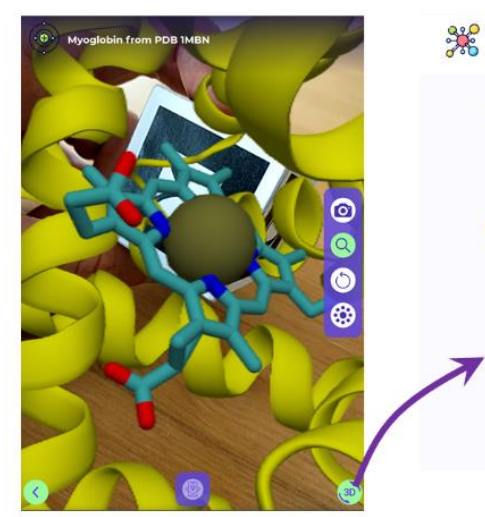

$\mathbf{F}$

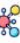
IMBN

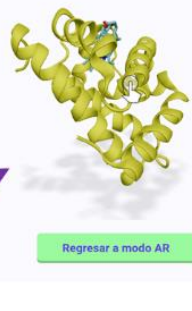

B

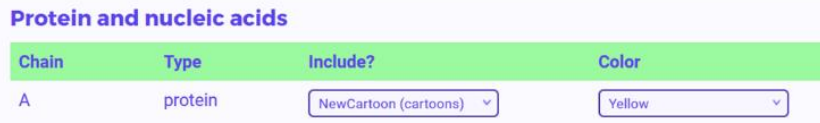

Ligands

Chain Molecule Residue number Include? Color Notes

A 0 .

A HEM

A HEM

$\mathrm{HOH}$

WAT

TIP

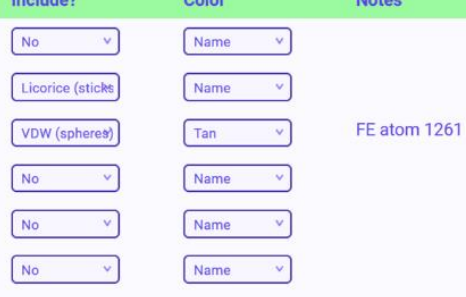

Build VMD script

D

\section{Your AR project is ready!}

MoleculARweb [noreply@molecularweb.epfl.ch] $\mathrm{Hi}$ !

Here's the url for your AR project:

Panel E-G

https://molecularweb.epfl.ch/pdb2ar/mbnvr

Here's the url for your project in VR:

https://molecularweb.epfl.ch/.pdb2ar/mbnvr/vr

Here's the 3D model if you want to download it:

https://molecularweb.epfl.ch/pdb2ar/mbnvr/model.zip

Enjoy!

G

H

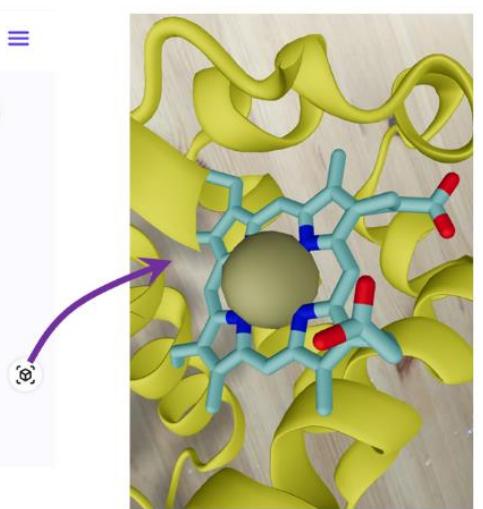

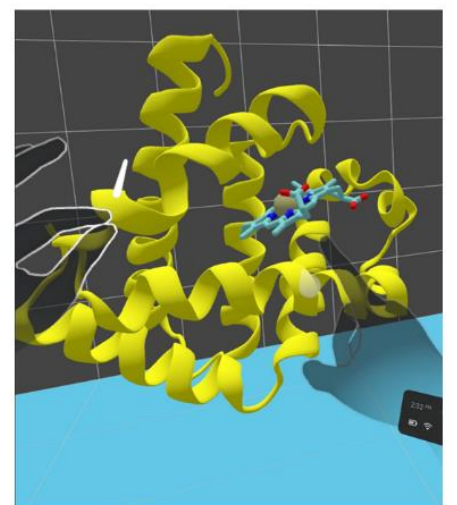

Figure 2. Creating a model from PDB $1 M B N$ (myoglobin) to show its overall fold as cartoons, heme group as sticks and iron ion as a sphere. In (A) the user fetches of copy-pastes the PDB file and clicks "Detect molecules"; in (B) it choses what representations and colors to use and clicks "Build VMD script" to the get the VMD script shown in (C). Users familiar with VMD can optionally edit this script. After providing a title and email address the user clicks "Submit" and after a few seconds will receive an email with a unique link to display the AR experience (D). Opening the link in the email leads first to visualization with a cube marker (https://molecularweb.epfl.ch/assets/markers/allmarkers.pdf) that works in phones, laptops or computers (E). Clicking on the 3D button opens the white background view (F), that also works on all devices and where users move the object with the finger or mouse. Then clicking on the last button leads to the immersive AR mode where the object sits on flat surfaces, without any markers, on smartphones and tablets (G). We leave this specific example on myoglobin ready to view at https://molecularweb.epfl.ch/pdb2ar/1mbnnuevo323. Notice that AR with 
markers works in all modern browsers and operative systems on smartphones, tablets and desktop computers (user needs to enable webcam use when prompted for the first time); while AR without markers works only in Chrome (best if version 92 or higher) on WebXR-enabled smartphones. $(\mathrm{H})$ The same 3D model as in E-G but seen in the web browser of an Oculus Quest 2 device, where the user can manipulate the molecule with its bare hands. This example is available at https://molecularweb.epfl.ch/pdb2ar/1mbnnuevo323/vr and it does run on smartphones, tablets and computers too.

\section{Input mode 2: VMD-generated Wavefront objects}

If the user is familiar enough with VMD to prepare its own models as Wavefront objects (pairs of OBJ-MTL files), these can be submitted directly together with a title and an email address to let the system build the AR displays. The user then receives an email (Figure 3A) with a link to the AR views much like with the normal PDB-based input mode (examples in Figure 3B-H) and with a separate link for high-end VR in headsets with WebXR-compatible browsers (examples in Figure 4).

This input mode has two advantages. For PDB files, it allows finer control than what input mode 1 offers (although mode 1 is already quite complete and can be extended by editing the VMD script before submitting to the website, at the stage shown in Figure 2C). But most importantly, input mode 2 extends the range of applications as it allows users to build in their local computers 3D scenes from molecules other than proteins or nucleic acids, and even from objects other than atomic coordinates such as isosurfaces or meshes representing orbitals or electron densities. As examples, Figure 3B shows a membrane with its lipids represented as sticks; Figure $3 \mathrm{C}$ shows a $\mathrm{T} 4$ bacteriophage rendered as an isosurface from an experimental Cryo-electron tomography map ${ }^{7}$; Figure 3D shows a composition for a membrane-embedded dopamine receptor bound to a transmembrane $\mathrm{G}$ protein and with a ligand bound ${ }^{8}$; Figure $3 \mathrm{E}$ shows an aerolysin molecule inserted inside a membrane, acting as a ion-conducting nanopore; ${ }^{9}$ Figure $3 \mathrm{~F}$ shows a cryotomography-based 3D reconstruction of the SARS-Cov2 viral particle, with even Spike and ribonucleoproteins resolved; ${ }^{10}$ Figure $3 \mathrm{G}$ shows the active site of carbonic anhydrase with a ligand bound where only selected residues are displayed as sticks; and Figure $3 \mathrm{H}$ shows a liposome patch rendered from a coarse-grained model using a combination of Quick Surface and VDW representations. 
A
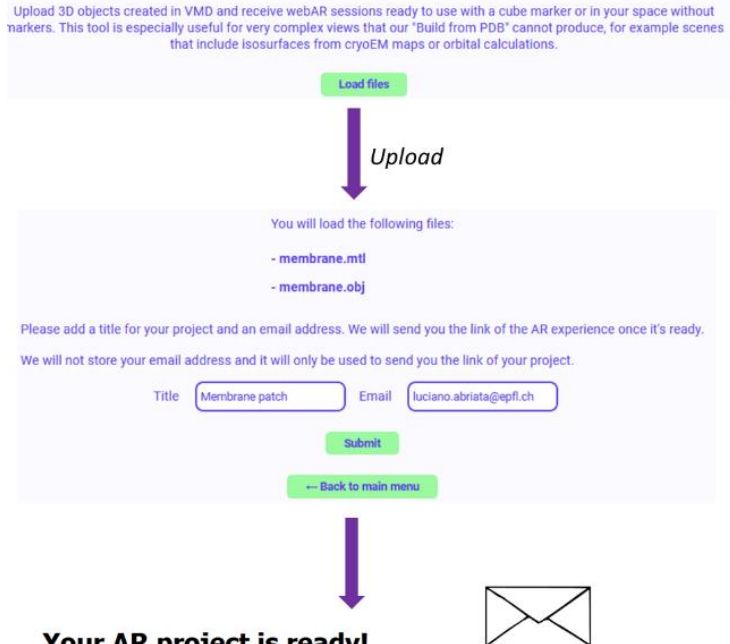

MoleculARweb $\vec{\otimes}$ [noreply@molecularweb.epfl.ch]

https://molecularweb.epfl.ch/pdb2ar/membranepatch
B

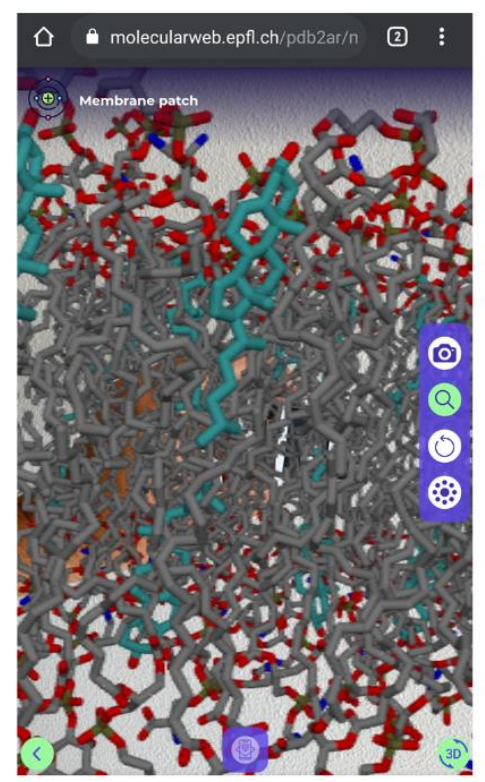

C

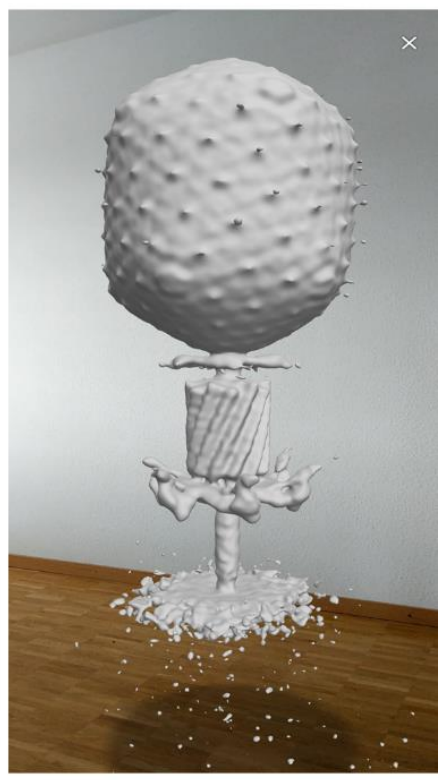

E

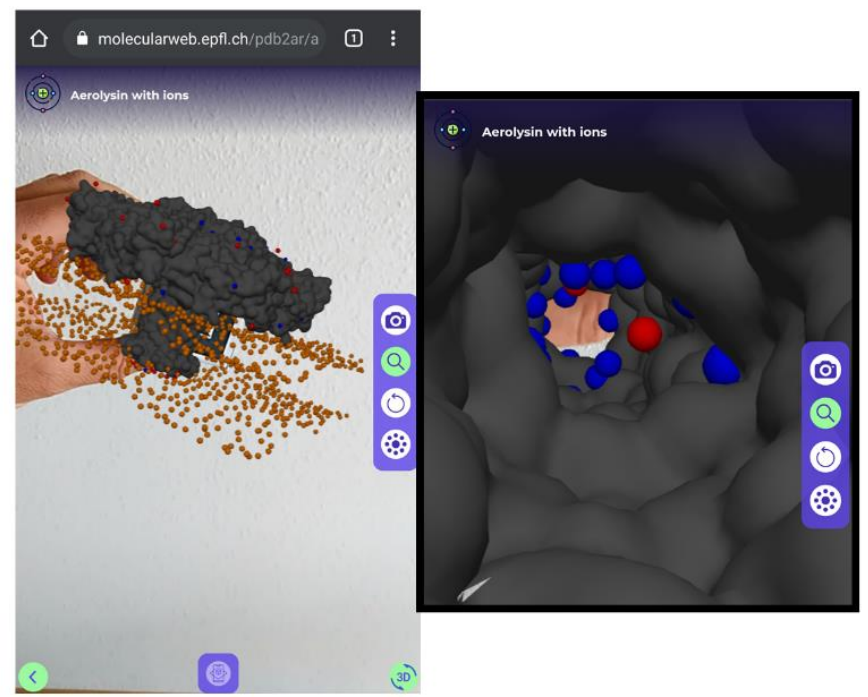

G

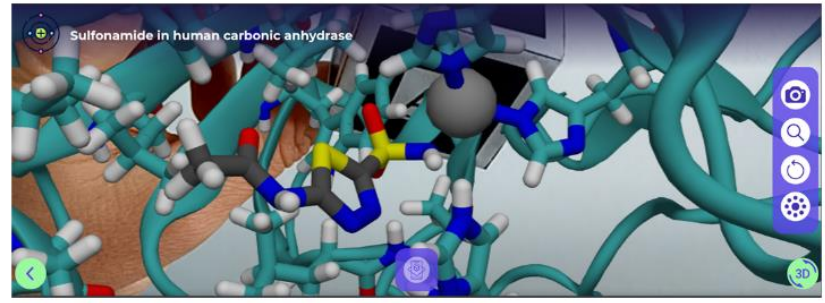

H

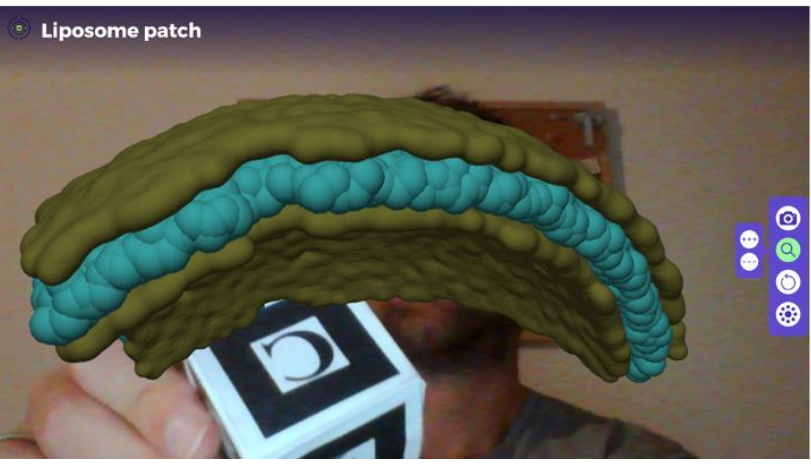

F

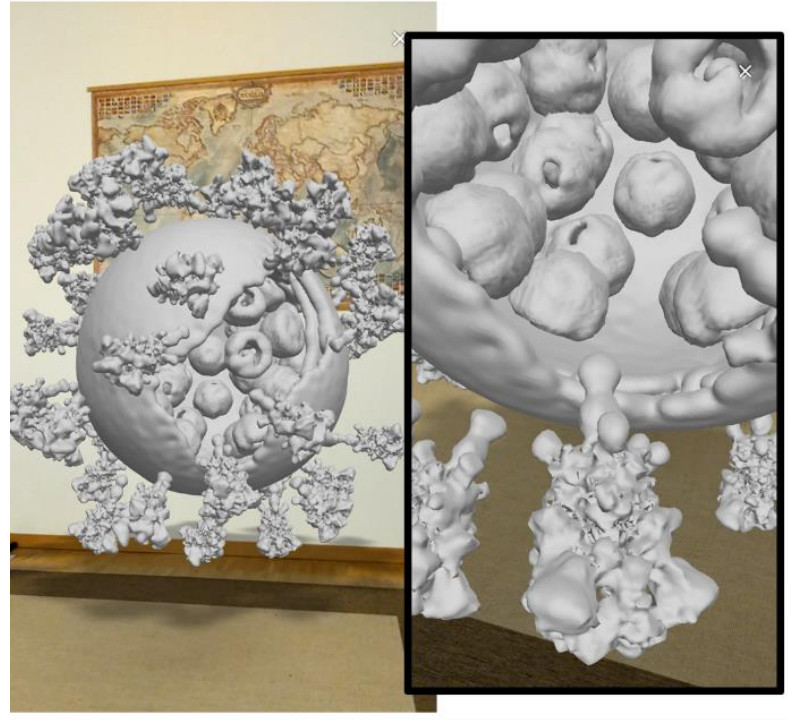

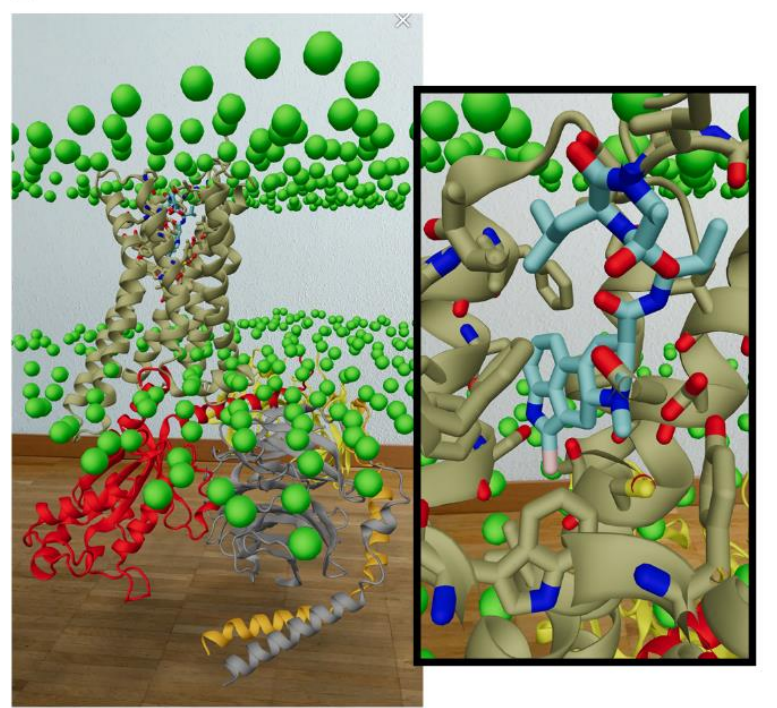


Figure 3. Creating WebAR views from VMD scenes. (A) Pipeline: the user uploads Wavefront files (one .OBJ and one .MTL file) generated in VMD and provides a title and an email address. From this point onwards, the procedure is the same as for Input mode 1 detailed in Figures 1 and 2: the user receives an email with a link to a first activity where the object is displayed on the cube marker, and clicking on the "3D" button switches to the markerless modes. We show three example systems. (B) A patch of eukaryotic cell membrane modeled with CHARMM-GUI ${ }^{11}$ with phospholipids in grey and cholesterol in cyan, here seen on a cube marker in a smartphone (example accessible at https://molecularweb.epfl.ch/pdb2ar/membranepatch/). (C) T4 bacteriophage rendered as isosurface from Cryoelectron tomography map (EMDB $6082^{7}$ ), accessible at https://molecularweb.epfl.ch/pdb2ar/t4bacteriophage/. (D) A dopamine receptor bound to a G protein and a ligand, seen without markers, from PDB ID 6VMS. ${ }^{8}$ In the inset the user moved into the ligand binding site. Example available at https://molecularweb.epfl.ch/pdb2ar/gpcr/. (E) An aerolysin molecule inserted in a membrane, snapshot from a simulation of ion conductance across the protein used as a nanopore ${ }^{9}$ (blue spheres are $\mathrm{K}^{+}$ions and red spheres are $\mathrm{Cl}^{-}$ions). Example accessible at https://molecularweb.epfl.ch/pdb2ar/aerolysinwithions/. (F) 3D shape of the SARS-Cov2 virus assembled from cryoelectron tomography and subtomogram averaging (EMDB $30430^{10}$ ). Notice the ribonucleoparticles inside the virus and the Spike proteins exposed on its surface. Example accessible at https://molecularweb.epfl.ch/pdb2ar/sarscov2experimental/. (G) Crystal structure of a sulfonamide-bound human carbonic anhydrase (PDB 1AZM, hydrogens added ${ }^{12}$ ) where only the drug and the protein residues of its binding pocket are shown as sticks, and the enzyme's zinc ion as a sphere. This screenshot was taken on a smartphone in landscape orientation, with the system on a cube marker. See at https://molecularweb.epfl.ch/pdb2ar/sulfonamideinhumancarbonicanhydrase/. (H) A liposome patch generated with CHARMM-GUI ${ }^{11}$ with its coarse-grained MARTINI builder. The polar heads are represented as smooth surfaces in tan color and the hydrophobic portion of the bilayer is completed as cyan coarse-grained spheres. This view is in a laptop computer using the cube marker. Example at https://molecularweb.epfl.ch/pdb2ar/liposomepatch/. Notice that AR with markers works in all modern browsers and operative systems on smartphones, tablets and desktop computers (user needs to enable webcam use when prompted for the first time); while AR without markers works only in Chrome (best if version 92 or higher) on WebXR-enabled smartphones.

Virtually any scene created with solid objects in VMD can be exported as a Wavefront object (File $\rightarrow$ Render, choose Wavefront, path and click "Start Rendering") and then seen in AR or VR through this tool. By "solid objects" we mean that line objects are not exported by VMD, at least as of version 1.9.4. Adding to the AR examples in Figure 3, Figure 4 shows screenshots taken inside a VR device on four example systems exported right from VMD: a piece of double-stranded DNA (Figure 4A), a molecule with an inhibitor bound (Figure 4B where the user is seen on the right pointing at a feature of the structure, example video in the figure caption), a cryo-electron map with a protein fit inside (Figure 4C), and a viral particle reconstructed in 3D by cryo-electron tomography (Figure 4D, two views). 
A

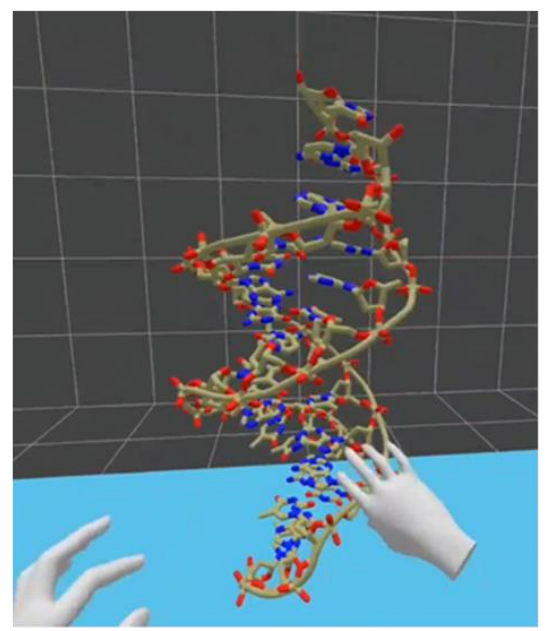

C

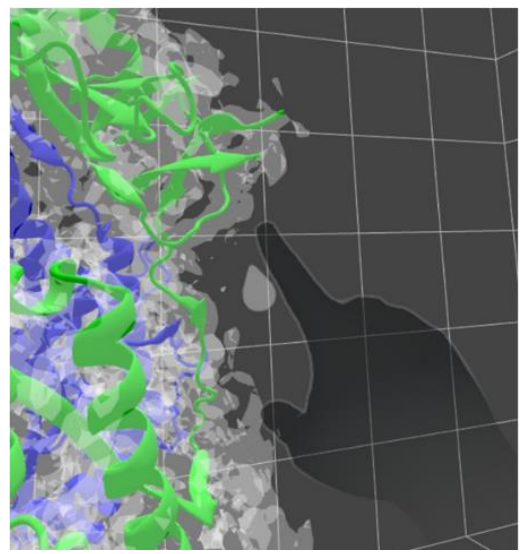

B

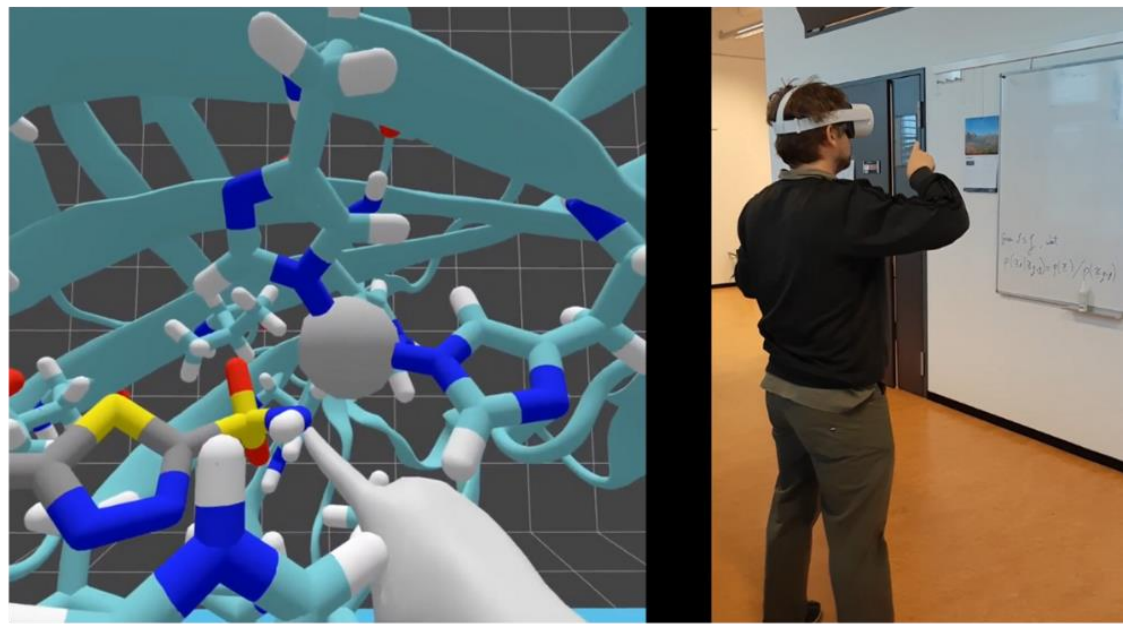

D

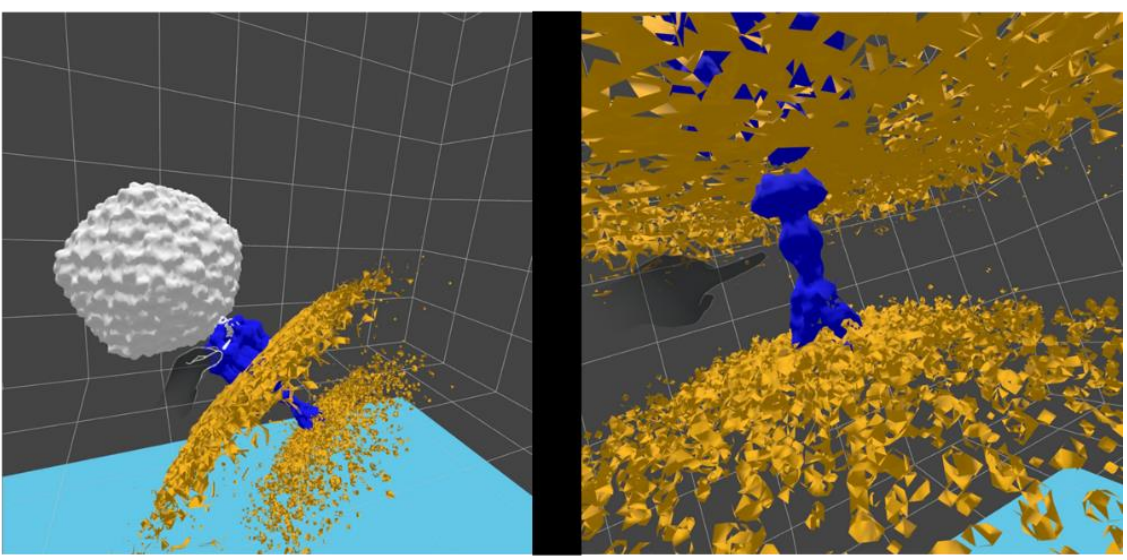

Figure 4. 3D views exported from VMD and running on the web browser of an Oculus Quest 2 VR device. The pipeline is the same as show in Figure 3A, but the user must follow the VR link. (A) A piece of double-stranded DNA molecule, the the user is grabbing around its major groove. (B) The same model as in Figure $3 G$ but in immersive VR; the user is seen on the right pointing at the small molecule bound to the protein's zinc ion. For a video of this, follow https://vimeo.com/651088983 . (C) A structure fit inside a density isosurface from a cryo-electron microscopy map. (D) Two views of a bacteriophage model resolved by cryo-electron tomography, where it was "caught" with its needle (blue) inserted through the two bacterial membranes.

\section{Final remarks}

We have presented here a new tool to help users create their own AR or VR views from plain PDB files containing atomic coordinates or from virtual objects or scenes exported from the VMD program. The tool is intended for educators and science communicators, and is open for free use without registration. Being webbased, they are very easy to deploy, distribute, and use; see for example the blog entries at 
Importantly, AR with markers works in all modern browsers and operating systems on smartphones, tablets and desktop computers (the user needs to allow webcam use when prompted for the first time); while AR without markers works only in Chrome (best if version 92 or higher) on WebXR-enabled smartphones and tablets. The immersive VR views work only on VR headsets with WebXR-enabled web browsers, which are becoming the standard as major academic and industrial players develop the software and hardware for the Metaverse.

We intend to keep the user-created content available for at least one month, but we reserve the right to shorten this time if required. When starting from PDB files, users can anyway always download the created OBJ+MTL files for future use in the server by going directly through input mode 2 . We also reserve the right to select user-uploaded VR scenes of especial interest to use them as activities in the main moleculARweb website and for website promotion.

We conclude by reminding educators and students to please fill the surveys at https://molecularweb.epfl.ch/pages/feedback.html after having worked with the activities presented here or with any other activities of the moleculARweb site. This information is crucial to keep the website growing, to learn about how educators use the AR activities, and to learn to what extent AR and VR actually help students to learn.

\section{Methods}

The tools presented here rely on the same technologies used for our MoleculARweb ${ }^{1}$ site. Briefly, AR views are rendered entirely in the user's device through content written entirely in HTML5, CSS and JavaScript, ${ }^{13,14}$ exploiting several free, open-source libraries especially Three.js, AR.js, jsartoolkit, and ModelViewer. ${ }^{15}$ The backend is built using node.js, express.js, nodemailer and multer to handle file upload, run VMD scripts, generate the output web pages, and deliver the outputs to users by e-mail. All the code behind these tools is 
available at GitHub: $\underline{\text { https://github.com/fcor/molecular-mirror-app }} \quad$ (client) and

https://github.com/fcor/molecular-mirror-backend (backend).

\section{Acknowledgements}

This work was funded by SNF Agora grant CRARP2_202370 to LAA.

\section{References}

(1) Cortés Rodríguez, F.; Frattini, G.; Krapp, L. F.; Martinez-Hung, H.; Moreno, D. M.; Roldán, M.; Salomón, J.; Stemkoski, L.; Traeger, S.; Dal Peraro, M. MoleculARweb: A Web Site for Chemistry and Structural Biology Education through Interactive Augmented Reality out of the Box in Commodity Devices. J. Chem. Educ. 2021.

(2) Fernandes, H. S.; Cerqueira, N. M.; Sousa, S. F. Developing and Using BioSIMAR, an Augmented Reality Program to Visualize and Learn about Chemical Structures in a Virtual Environment on Any InternetConnected Device; ACS Publications, 2021.

(3) Abriata, L. A. How Technologies Assist Science Learning at Home during the Covid19 Pandemic. DNA Cell Biol. 2021, $X$.

(4) Humphrey, W.; Dalke, A.; Schulten, K. VMD: Visual Molecular Dynamics. J. Mol. Graph. 1996, 14 (1), 33-38.

(5) Rodríguez, F. C.; Dal Peraro, M.; Abriata, L. A. Democratizing Interactive, Immersive Experiences for Science Education with WebXR. Nat. Comput. Sci. 2021, 1 (10), 631-632.

(6) Watson, H. C. The Stereochemistry of the Protein Myoglobin. Prog Stereochem 1969, 4 (299), 5.

(7) Hu, B.; Margolin, W.; Molineux, I. J.; Liu, J. Structural Remodeling of Bacteriophage T4 and Host Membranes during Infection Initiation. Proc. Natl. Acad. Sci. 2015, 112 (35), E4919-E4928.

(8) Yin, J.; Chen, K.-Y. M.; Clark, M. J.; Hijazi, M.; Kumari, P.; Bai, X.; Sunahara, R. K.; Barth, P.; Rosenbaum, D. M. Structure of a D2 Dopamine Receptor-G-Protein Complex in a Lipid Membrane. Nature 2020, 584 (7819), 125-129.

(9) Cirauqui, N.; Abriata, L. A.; van der Goot, F. G.; Dal Peraro, M. Structural, Physicochemical and Dynamic Features Conserved within the Aerolysin Pore-Forming Toxin Family. Sci. Rep. 2017, 7 (1), 13932. https://doi.org/10.1038/s41598-017-13714-4.

(10) Yao, H.; Song, Y.; Chen, Y.; Wu, N.; Xu, J.; Sun, C.; Zhang, J.; Weng, T.; Zhang, Z.; Wu, Z. Molecular Architecture of the SARS-CoV-2 Virus. Cell 2020, 183 (3), 730-738. e13.

(11) Jo, S.; Lim, J. B.; Klauda, J. B.; Im, W. CHARMM-GUI Membrane Builder for Mixed Bilayers and Its Application to Yeast Membranes. Biophys. J. 2009, 97 (1), 50-58. https://doi.org/10.1016/j.bpj.2009.04.013.

(12) Chakravarty, S.; Kannan, K. K. Drug-Protein Interactions: Refined Structures of Three Sulfonamide Drug Complexes of Human Carbonic Anhydrase I Enzyme. J. Mol. Biol. 1994, 243 (2), 298-309.

(13) Abriata, L. A.; Rodrigues, J. P. G. L. M.; Salathé, M.; Patiny, L. Augmenting Research, Education, and Outreach with Client-Side Web Programming. Trends Biotechnol. 2018, 36 (5), 473-476. https://doi.org/10.1016/j.tibtech.2017.11.009.

(14) Abriata, L. A. Web Apps Come of Age for Molecular Sciences. Informatics 2017, 4 (3), 28. 
(15) Abriata, L. A. Building Blocks for Commodity Augmented Reality-Based Molecular Visualization and Modeling in Web Browsers. PeerJ Comput. Sci. 2020, 6, e260. 